\title{
Diferenciais de mortalidade por sexo no município de São Paulo em 2005 e 2016: contribuição dos grupos etários e das principais causas de óbito
}

\author{
Pamila Cristina Lima Siviero* \\ Larissa Gonçalves de Souza** \\ Carla Jorge Machado***
}

O objetivo deste trabalho é analisar o diferencial de mortalidade por sexo no município de São Paulo, em 2005 e 2016, por meio de análises dos níveis e padrões do diferencial, além de examinar a contribuição dos diversos grupos etários e das principais causas de óbito. Os indicadores calculados foram o diferencial da esperança de vida ao nascer, a razão de sexo entre as taxas específicas de mortalidade e a contribuição dos grupos etários e das causas de morte para o diferencial. Os resultados evidenciam que o maior ganho dos homens em esperança de vida ao nascer contribuiu para a redução de 1,30 ano do diferencial entre os dois anos estudados. Ademais, apesar das elevadas razões de sexo entre as taxas específicas de mortalidade dos jovens, os idosos foram os que mais contribuíram para explicar a vantagem feminina na mortalidade. Por último, as doenças do aparelho circulatório, neoplasias e causas externas foram responsáveis pelas maiores contribuições para o diferencial. No entanto, a análise do padrão de causas de óbito em conjunto com o padrão etário mostra que, para os idosos, cuja participação é maior para o diferencial, as doenças do aparelho circulatório, neoplasias e doenças do aparelho respiratório foram mais decisivas na formação do diferencial.

Palavras-chave: Diferencial de mortalidade por sexo. Decomposição. Causas de óbito.

\footnotetext{
* Universidade Federal de Alfenas (Unifal), Varginha-MG, Brasil (pclsiviero@gmail.com; https://orcid.org/0000-00032042-812X).

** Universidade Federal de Alfenas (Unifal), Varginha-MG, Brasil (gso.larissa@gmail.com; https://orcid.org/0000-00033536-3912).

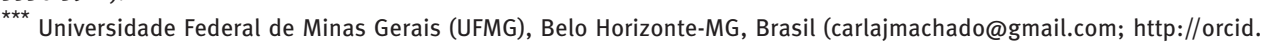
org/0000-0002-6871-0709).
} 


\section{Introdução}

O declínio histórico dos níveis de mortalidade, observado em todos os países que experimentam o processo de transição demográfica, resulta no aumento da esperança de vida das populações. No entanto, mesmo diante de tantos ganhos, este processo tem como consequência alguns diferenciais (SUNDBERG et al., 2018), dos quais destaca-se o diferencial entre homens e mulheres. Segundo Glei e Horiuch (2007), as diferenças na mortalidade entre os sexos, caracterizadas por maior expectativa de vida das mulheres, frequentemente superam o hiato entre outros grupos populacionais, como, por exemplo, entre raças/etnias.

O sexo é um dos determinantes mais importantes da inserção dos indivíduos na sociedade. Tal inserção é construída durante a vida, por meio de valores culturais e sociais. As diferenças observadas entre os sexos muitas vezes determinam estilos de vida distintos e afetam a maneira e o momento que homens e mulheres adoecem e, portanto, as condições que os levam à morte (CHOR et al., 1992; CASE; PAXSON, 2005). 0 sexo é também um dos critérios mais evidentes de distinção genética entre indivíduos da mesma espécie. Para espécies humanas, entretanto, ser homem ou mulher está associado também a diferenças psicológicas, sociais, econômicas, entre outras, de modo que o aspecto genético das diferenças observadas na mortalidade pode ser apenas marginal (VALLIN, 1983, 2004; KALBEN, 2000; LUY; WEGNER-SIEGMUND, 2015).

Na maioria das populações, os homens experimentam maior mortalidade do que as mulheres, a cada idade, exceto em alguns países situados na Ásia (LANGFORD; STOREY, 1993; PATTON et al., 2009). A magnitude da diferença varia de acordo com condições sociais, econômicas e ambientais. Nos países desenvolvidos, durante grande parte do século XX, a mortalidade total declinou, o padrão de causas de óbito mudou de uma maior representatividade das doenças transmissíveis para as não transmissíveis e causas externas e a desvantagem masculina continuou a crescer. Entretanto, nessas nações, desde 1970, o diferencial entre os sexos na esperança de vida ao nascer vem se reduzindo sistematicamente (GLEI, 2005; TROVATO, 2005; PRESTON; WANG, 2006; GLEI; HORIUCH, 2007; ROGERS et al., 2010; SUNDBERG et al., 2018).

Para os países em desenvolvimento e menos desenvolvidos, a situação é menos clara, sobretudo devido à ausência de informações confiáveis (LANGFORD; STOREY, 1993; WISSER; VAUPEL, 2014). Mesmo com essa limitação, alguns autores observaram que o diferencial na esperança de vida ao nascer entre os sexos também aumentou com o declínio da mortalidade (OHADIKE, 1983; VALLIN, 1983; SHRESTHA, 2000; GEE, 2002; UNITED NATIONS, 2005, 2007, 2017; CHISUMPA; ODIMEGWU, 2018), embora seja menor do que aquele registrado nos países mais desenvolvidos (GEE, 2002; SHRESTHA, 2000; UNITED NATIONS, 2005, 2007, 2017) e ainda menor nas regiões menos desenvolvidas, que experimentam condições socioeconômicas e de saúde muito desfavoráveis (UNITED NATIONS, 2005, 2007, 2017; CHISUMPA; ODIMEGWU, 2018). 
O Brasil seguiu a tendência mundial, uma vez que as mulheres apresentaram maior esperança de vida do que os homens durante a segunda metade do século XX e houve uma rápida ampliação da diferença entre as esperanças de vida feminina e masculina (SIMÕES, 2002). Especificamente para o município de São Paulo, estudo mostrou que o comportamento desse diferencial assemelhou-se àquele observado nos países desenvolvidos: aumentou ao longo do século XX e vem diminuindo desde os anos 1990 (SIVIERO et al., 2011).

Apesar de sua relevância, no Brasil há poucos estudos que investigam as disparidades na mortalidade entre os sexos no nível nacional, de suas unidades federativas e municípios. Nesse sentido, a análise temporal das variações do diferencial de mortalidade entre os sexos, assim como do paradoxo entre saúde e sobrevivência, nas idades avançadas, pode trazer resultados importantes para a compreensão desse processo. A estimativa de mortalidade e a avaliação de seus diferenciais podem auxiliar na formulação de políticas de saúde direcionadas à redução das desigualdades em grupos populacionais específicos, bem como estimar uma média da duração dos cuidados necessários com determinada população, por meio dos anos vividos com ou sem saúde (CAMARGOS; GONZAGA, 2015).

Nesse contexto, o objetivo do presente trabalho é analisar o diferencial de mortalidade entre os sexos no município de São Paulo, em 2005 e 2016, por meio de análises dos níveis e padrões do diferencial, além de examinar a contribuição dos diversos grupos etários e das causas de óbito para o diferencial na esperança de vida ao nascer entre homens e mulheres.

\section{Diferenciais de mortalidade por sexo}

A mortalidade masculina é superior à feminina em todos os grupos etários e na maioria dos países, assim como a expectativa de vida ao nascer e em outras idades geralmente é maior entre as mulheres (VALLIN, 2004; WISSER; VAUPEL, 2014; SUNDBERG et al., 2018). As poucas exceções ocorrem em alguns países situados no sul do continente Asiático, onde tem sido relatado que as condições de vida e saúde das mulheres são inferiores às dos homens (KALBEN, 2000; PATTON et al., 2009).

Acredita-se que as razões para essas diferenças de mortalidade e incapacidade tenham origens biológicas e não biológicas (VALLIN, 2004; SUNDBERG et al., 2018; YOKOTA et al., 2018). Enquanto as primeiras estão relacionadas a fatores endógenos (biológicos e genéticos), portanto, além do controle humano, as não biológicas referem-se a fatores exógenos (sociais, comportamentais e ambientais), influenciados direta ou indiretamente pela ação humana (VALLIN, 2004).

Em termos biológicos, parte dos diferenciais de mortalidade e de incapacidade entre os sexos está relacionada a causas hormonais, autoimunes e genéticas (VALLIN, 2004; OKSUZYAN et al., 2008). Assim, os diferenciais de mortalidade entre os sexos estariam relacionados à diferença genética entre homens e mulheres, dada pelo segundo cromossomo Y e X, respectivamente (VALLIN, 2004). Além disso, os hormônios femininos também desempenham papel importante para garantir maior imunidade e proteger as mulheres 
contra determinadas doenças, como, por exemplo, a cardíaca (KALBEN, 2000; LUY, 2003; VALLIN, 2004). Apesar da relevância dos fatores biológicos, estudos atribuíram a maior parte da diferença entre as expectativas de vida a fatores não biológicos (OKSUZYAN et al., 2008; LUY; WEGNER-SIEGMUND, 2015; LUY; GAST, 2014; SUNDBERG et al., 2018).

As perspectivas sociais e comportamentais tendem a enfatizar os diferentes estilos de vida, cuidados com saúde e fatores socioeconômicos (ROGERS et al., 2010; LUY; GAST, 2014; SUNDBERG et al., 2018). Os defensores dessas hipóteses argumentam que a sociedade e o aspecto cultural conduzem os homens a um estilo de vida mais arriscado. Dessa maneira, os comportamentos relacionados a consumo de álcool e tabaco, hábitos alimentares irregulares, riscos ocupacionais e direção imprudente são vistos como fundamentais para explicar os diferenciais de mortalidade entre homens e mulheres (KALBEN, 2000; LUY, 2003; ROGERS et al., 2010; SUNDBERG et al., 2018). Outros aspectos importantes dizem respeito à maior disposição das mulheres em cuidar da saúde e à melhor percepção de sintomas de doenças (KALBEN, 2000; LUY, 2003).

Em relação ao comportamento da vantagem feminina na mortalidade, os países industrializados experimentaram uma variação espacial e temporal ao longo da transição da mortalidade. No final do século XIX, o hiato na expectativa de vida ao nascer entre os sexos era relativamente pequeno, sendo inferior a três anos (GLEI, 2005; TROVATO, 2005). No entanto, essa situação se alterou ao longo de grande parte do século $\mathrm{XX}$, quando o diferencial cresceu rapidamente em favor das mulheres (TROVATO, 2005).

Dentre as causas apontadas para explicar a ampliação do diferencial, encontram-se o consumo de tabaco, que era socialmente aceito como um hábito comum entre os homens, na primeira metade do século XX (PAMPEL, 2002, 2005, 2006), e o declínio mais lento da mortalidade para o sexo masculino relativamente ao feminino (GLEl, 2005). Além disso, de acordo com Glei (2005), a ampliação do diferencial de mortalidade entre os sexos ocorreu num contexto de importantes mudanças demográficas, como os aumentos substanciais de expectativa de vida e a concentração dos óbitos nas idades mais avançadas.

No entanto, durante as décadas de 1970 e 1980, os países experimentaram uma reversão desta tendência e o diferencial de mortalidade entre os sexos começou a se reduzir em alguns países desenvolvidos, como, por exemplo, naqueles de origem anglo-saxônica (GLEI, 2005; WISSER; VAUPEL, 2014). Desde então, a diferença na expectativa de vida entre homens e mulheres tem diminuído continuamente na maioria dos países desenvolvidos (GLEI; HORIUCH, 2007). Diversos fatores são adotados para explicar a redução do hiato entre a expectativa de vida ao nascer feminina e masculina, dentre os quais está a mudança nos padrões do uso do tabaco, especificamente um aumento do consumo entre as mulheres (PAMPEL, 2005). No entanto, Luy e Wegner-Siegmund (2015) sugerem que o tabagismo não deve ser considerado a principal causa da retração no diferencial por sexo na longevidade, uma vez que o seu impacto varia muito entre populações. A importância de outros fatores não biológicos, além do comportamento tabagista, também foi observada por Sundberg et al. (2018). 
Segundo Vallin (2004), a trajetória do diferencial é caracterizada pelo seguinte comportamento: quanto mais se eleva a expectativa de vida mais se aumenta o diferencial; no entanto, quanto maior a expectativa de vida, menor é o diferencial. Além disso, após o início de redução do diferencial, dois comportamentos adicionais têm sido observados: 0 impacto crescente da mortalidade dos idosos na diferença entre os sexos de expectativa de vida ao nascer; e a participação das doenças circulatórias na diminuição desse diferencial (SUNDBERG et al., 2018).

A maior contribuição da população idosa para o hiato na expectativa de vida ao nascer pode estar relacionada ao aumento da expectativa de vida na velhice (SUNDBERG et al., 2018) e à maior concentração dos óbitos nas idades mais avançadas. A redução da mortalidade por doenças circulatórias nos países desenvolvidos, por sua vez, tem explicado a maior parte do aumento da expectativa de vida e da diminuição do hiato na expectativa de vida entre homens e mulheres (LE et al., 2015; SUNDBERG et al., 2018).

0 comportamento do diferencial no Brasil assemelhou-se àquele observado nos países desenvolvidos: rápida ampliação da diferença entre as esperanças de vida entre os sexos, ao longo da segunda metade do século XX, seguida por uma redução do hiato (BELON; LIMA; BARROS, 2014). 0 mesmo foi verificado para o município de São Paulo, no qual o diferencial cresceu ao longo do século XX e vem diminuindo desde os anos 1990 (SIVIERO, 2009).

\section{Materiais e métodos}

Neste trabalho foram utilizados dados de óbitos e população do município de São Paulo, em 2005 e 2016. 0 período de análise foi escolhido por ser o mais recente com informações disponíveis. Os dados de óbitos desagregados por sexo, grupos etários e causas de morte, de acordo com a décima revisão da Classificação Internacional de Doenças (CID 10), são provenientes do Sistema de Informação sobre Mortalidade (SIM) e foram coletados no endereço eletrônico do Departamento de Informática do Sistema Único de Saúde (Datasus). Com o intuito de reduzir o efeito de possíveis flutuações aleatórias no número de óbitos por idade, foram consideradas, para 2005, a média dos óbitos ocorridos em 2004, 2005 e 2006, e, para 2016, a média de 2015, 2016 e 2017. As informações sobre a população residente, desagregadas por sexo e grupos etários, são projeções populacionais disponibilizadas pela Fundação Seade. 0 município de São Paulo foi escolhido devido à boa qualidade do registro de óbitos, com cobertura praticamente completa desde 1940 (SIVIERO et al., 2011).

Para a análise dos níveis e padrões do diferencial de mortalidade entre os sexos, foram calculados os seguintes indicadores: diferencial da esperança de vida ao nascer; razão de sexo entre as taxas específicas de mortalidade; e a contribuição dos grupos etários e das causas de morte para o diferencial.

Para a construção dos indicadores utilizados, foram calculadas as taxas específicas de mortalidade de 2005 e 2016, por grupos quinquenais de idade e pelos grupos de 
causas de óbito selecionados para o estudo, quais sejam: Cap. I - Algumas doenças infecciosas e parasitárias; Cap. II - Neoplasmas; Cap. IX - Doenças do aparelho circulatório; Cap. X - Doenças do aparelho respiratório; Cap. XII - Doenças do aparelho digestivo; Cap. XX - Causas externas; e Demais causas - grupo que contempla os demais capítulos da CID 10. As expectativas de vida foram obtidas por meio das tábuas de mortalidade para as populações de homens e mulheres (Tabelas A1 a A4 do Anexo). Essas tábuas foram construídas considerando-se que uma coorte hipotética de recém-nascidos vivenciou a experiência de mortalidade da população do município de São Paulo nos anos estudados (CARVALHO et al., 1994; PRESTON et al., 2001).

0 hiato na esperança de vida ao nascer foi obtido por meio da diferença entre a esperança de vida ao nascer das mulheres e dos homens. 0 resultado permite analisar a tendência dos níveis do diferencial de mortalidade por sexo. Em seguida, para verificar o padrão etário do diferencial, foi calculada a razão de sexo entre taxas específicas de mortalidade. Essa medida mostra a desvantagem na mortalidade de um sexo em relação ao outro (WISSER; VAUPEL, 2014). A razão maior que 1 sugere que o risco experimentado pelos homens de um grupo etário é maior do que o das mulheres do mesmo grupo. 0 resultado inferior a 1 indica desvantagem feminina na mortalidade e igual a 1 sugere igualdade no risco de morte.

A razão de sexo entre as taxas específicas de mortalidade é uma medida de risco relativo que não traz informação sobre a magnitude das taxas de mortalidade experimentadas por cada grupo etário. Todavia, o diferencial na esperança de vida ao nascer é produzido e modificado ao longo dos anos como resultado de alterações nas taxas específicas de mortalidade, que nem sempre ocorrem na mesma proporção e sentido em todas as faixas etárias (GLEl, 2005). Portanto, para entender como os distintos grupos etários contribuem para o diferencial, foi adotado o método de decomposição da diferença de esperanças de vida entre os sexos, proposto pelas Nações Unidas (1982). Tal método decompõe a variação da esperança de vida ao nascer entre os sexos em um mesmo ano, a partir de três equações descritas a seguir.

Primeiro grupo etário:

${ }_{n} \Delta_{\mathrm{o}}=\left(e_{\mathrm{o}}^{f}-e_{\mathrm{o}}^{m}\right)-\left[\left(e_{\mathrm{o}+n}^{f}-e_{\mathrm{o}+n}^{m}\right) \cdot \frac{\left(l_{\mathrm{o}+n}^{f}+l_{\mathrm{o}+n}^{m}\right)}{2}\right]$.

Grupos etários intermediários:

${ }_{n} \Delta_{x}=\left[\left(e_{x}^{f}-e_{x}^{m}\right) \cdot \frac{\left(l_{x}^{f}+l_{x}^{m}\right)}{2}\right]-\left[\left(e_{x+n}^{f}-e_{x+n}^{m}\right) \cdot \frac{\left(l_{x+n}^{f}+l_{x+n}^{m}\right)}{2}\right]$.

Grupo etário aberto:

${ }_{\infty} \Delta_{x}=\left(e_{80}^{f}-e_{80}^{m}\right) \cdot \frac{\left(l_{80}^{f}+l_{80}^{m}\right)}{2}$

Onde: ${ }_{n} \Delta_{x}$ é a contribuição do grupo etário $x$ a $(x+n)$ para o hiato na esperança de vida ao nascer por sexo; $e_{x}$ é a esperança de vida à idade exata $x$; e $l_{x}$ é o número de sobreviventes à idade exata $x$. 
Além da análise por grupo etário, também é possível decompor a diferença na esperança de vida ao nascer por sexo, de acordo com as diferentes causas de óbito da população. Isso acontece porque a participação de cada causa no perfil de mortalidade geral pode contribuir para aumentar ou reduzir o diferencial (YANG et al., 2012). Para melhor visualização dos resultados, a decomposição foi calculada para os grupos etários de 0 a 19 anos, 20 a 49 anos e 60 anos e mais. As seguintes causas de óbito foram selecionadas, uma vez que são as mais prevalentes na população em estudo: doenças infecciosas e parasitárias; neoplasmas (neoplasias); doenças do aparelho circulatório; doenças do aparelho respiratório; doenças do aparelho digestivo; causas externas de morbidade e de mortalidade; e demais causas. As contribuições da causa de morte $j$ do grupo etário $x$ a $(x+n)$ para o hiato na esperança de vida ao nascer $\left({ }_{n} \Delta_{x}^{j}\right)$ foram encontradas por meio da seguinte equação:

${ }_{n} \Delta_{x}^{j}=\frac{{ }_{n}^{T E M_{x}^{j, f e m}}-{ }_{n} T E M_{x}^{j, \text { masc }}}{{ }_{n} T E M_{x}^{\text {fem }}-{ }_{n} T E M_{x}^{\text {masc }}} x_{n} \Delta_{x^{\prime}}$

Onde: ${ }_{n} T E M_{x}^{j}$ é a taxa específica de mortalidade entre as idades $x$ e $(x+n)$ pela causa $j$; e ${ }_{n} T E M_{x}$ é a taxa específica de mortalidade por todas as causas entre $x$ e $(x+n)$.

Logo, contribuições positivas indicam que a causa de morte $j$ elevou o diferencial, ou seja, a taxa específica de mortalidade pela causa analisada foi maior para os homens do que para as mulheres do grupo etário. Os valores negativos, por sua vez, indicam que a causa de óbito contribuiu para reduzir a diferença entre as esperanças de vida ao nascer feminina e masculina.

\section{Resultados}

Niveis do diferencial de mortalidade entre homens e mulheres

A Tabela 1 apresenta a evolução da esperança de vida ao nascer por sexo, no município de São Paulo, em 2005 e 2016. A tendência dos níveis de mortalidade e seus diferenciais podem ser analisados e comparados por meio deste indicador de longevidade.

TABELA 1

Evolução da esperança de vida ao nascer, por sexo

Município de São Paulo - 2005-2016

\begin{tabular}{cccc}
\hline Anos & Mulheres & Homens & Diferencial \\
\hline 2005 & 79,87 & 70,56 & 9,32 \\
2016 & 80,71 & 73,49 & 7,22 \\
\hline
\end{tabular}

Fonte: Sistema de Informações sobre Mortalidade; estimativas preliminares efetuadas em estudo patrocinado pela Ripsa; estimativas preliminares elaboradas pelo Ministério da Saúde/SVS/CGIAE.

Nota: Os dados foram obtidos no Datasus.

Em 2005, a esperança de vida ao nascer feminina era 79,87 anos, ao passo que a masculina correspondia a 70,56 anos. Dessa forma, o hiato na esperança de vida ao nascer 
entre os sexos era 9,32 anos. No entanto, entre 2005 e 2016 os homens experimentaram um incremento de 2,93 anos (4,1\%) em esperança de vida ao nascer, alcançando 73,49 anos. Em contrapartida, o ganho das mulheres no mesmo período foi de 0,84 ano $(1,1 \%)$, atingindo 80,71 anos. 0 maior incremento dos homens resultou na redução de 2,10 anos $(22,5 \%)$ do diferencial, que passou para 7,22 anos em 2016.

\section{Padrão etário do diferencial de mortalidade entre os sexos}

A análise da estrutura da mortalidade por idade ajuda a compreender a relação existente entre o diferencial no nível de mortalidade entre os sexos e a desvantagem masculina na mortalidade a cada idade. 0 Gráfico 1 apresenta as taxas específicas de mortalidade por idade (TEMs), de homens e mulheres, para o município de São Paulo nos anos em estudo. É possível observar que a mortalidade masculina é maior do que a feminina em todos os grupos etários. A despeito dessa diferença, há uma tendência de queda das taxas em todas as idades, para ambos os sexos, entre 2005 e 2016. No entanto, para os homens, tanto em 2005 quanto em 2016, nota-se aumento da mortalidade nas idades jovens, diferenciando o padrão da mortalidade masculina em relação à feminina (Gráfico 1).

GRÁFICO 1

Taxas específicas de mortalidade, por idade e sexo Município de São Paulo - 2005-2016

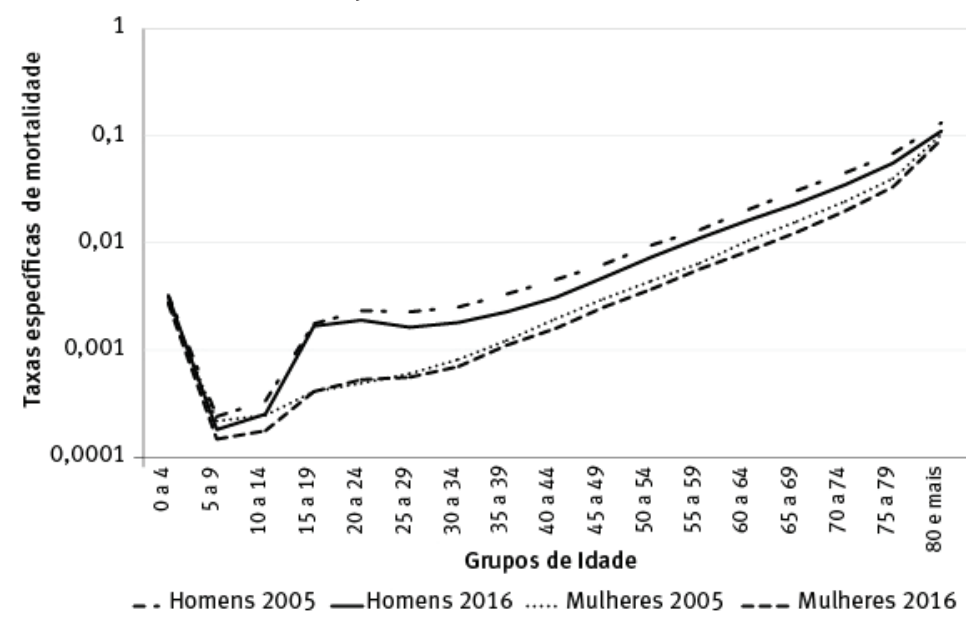

Fonte: Sistema de Informações sobre Mortalidade; estimativas preliminares efetuadas em estudo patrocinado pela Ripsa; estimativas preliminares elaboradas pelo Ministério da Saúde/SVS/CGIAE.

Visando interpretar de forma mais sistemática as diferenças observadas entre as TEMs, o Gráfico 2 apresenta as razões de sexo entre as taxas específicas de mortalidade para o município de São Paulo no período em estudo. A desvantagem masculina é evidenciada nos dois anos e em todos os grupos etários, uma vez que o indicador é sempre superior a 1. Para ambos os anos em estudo, o risco de morrer experimentado por um homem era maior especialmente nas idades mais jovens (entre 15 e 35 anos). Por exemplo, em 2005, 
entre as idades 20 e 24 anos, o risco de morrer experimentado pelos homens era 4,88 vezes o das mulheres, reduzindo para aproximadamente 2,0 vezes a partir dos 40 anos e menos de 1,5 vez a partir dos 75 anos. Em 2016, a estrutura do diferencial por idade se mantém semelhante, no entanto, o maior pico se desloca de 20 a 24 anos para 15 a 19 anos, sendo que o nível se torna menor em praticamente todos os grupos etários, com exceção do grupo de 5 a 9 anos de idade. As maiores quedas são observadas entre as idades de 20 a 40 anos (Gráfico 2).

\section{GRÁFICO 2}

Razões de sexo das taxas específicas de mortalidade Município de São Paulo - 2005-2016

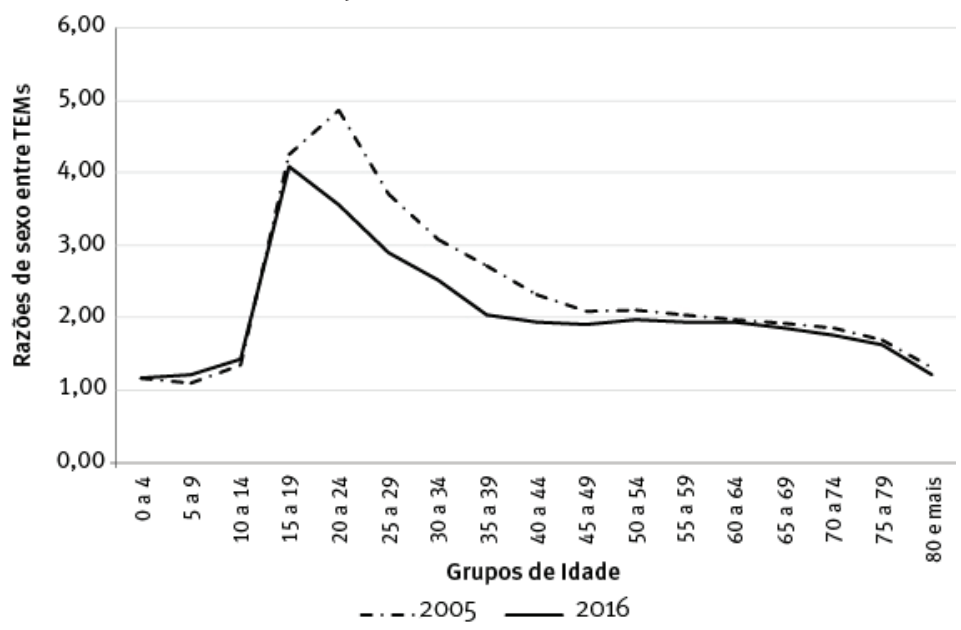

Fonte: Sistema de Informações sobre Mortalidade; estimativas preliminares efetuadas em estudo patrocinado pela Ripsa; estimativas preliminares elaboradas pelo Ministério da Saúde/SVS/CGIAE.

A Tabela 2 apresenta a contribuição das crianças e jovens, adultos e idosos para o hiato na esperança de vida ao nascer. Para facilitar a visualização dos resultados, optou-se por dividir a população em três grupos etários, sendo eles: 0 a 19 anos, 20 a 59 anos e 60 anos e mais. A análise dos resultados indica que mais de $90 \%$ do diferencial é explicado pelos adultos e idosos (20 a 59 anos e 60 anos e mais), sendo a participação das crianças e jovens ( 0 a 19 anos) pouco expressiva. Dentre adultos e idosos, a contribuição das idades avançadas (60 anos e mais) foi maior do que aquela experimentada pelos adultos, nos dois anos em estudo. Além disso, em termos relativos, os idosos aumentaram sua participação: em 2005 eles explicaram 49,08\% da vantagem feminina, aumentando para 53,87\% em 2016 (Tabela 2).

A análise por grupo etário quinquenal mostra que, com exceção da faixa de 75 a 79 anos, a partir dos 35 anos há uma tendência de aumento da contribuição absoluta e relativa para o diferencial, nos dois anos. Portanto, à medida que a idade avança, maior se torna a participação do grupo etário para explicar a vantagem das mulheres no diferencial de mortalidade entre os sexos. Dessa maneira, observa-se que, apesar da elevada 
razão entre as taxas específicas de mortalidade dos grupos etários jovens de 15 a 19 e de 20 a 24 anos, esse segmento populacional foi responsável por apenas aproximadamente $5 \%$ do hiato na esperança de vida ao nascer entre os sexos. Em relação ao decréscimo de 2,10 anos no nível do diferencial entre 2005 e 2016, os resultados mostram que todas as faixas etárias conduziram à redução do hiato ou não apresentaram variação (Tabela A5, do Anexo).

TABELA 2

Contribuição dos grupos etários para o diferencial na esperança de vida ao nascer por sexo Município de São Paulo - 2005-2016

\begin{tabular}{llrrrr}
\hline \multirow{2}{*}{$\begin{array}{c}\text { Grupo } \\
\text { etário }\end{array}$} & \multicolumn{2}{c}{ Contribuição } \\
\cline { 2 - 3 } (em anos) & $\mathbf{2 0 0 5}$ & $\mathbf{2 0 1 6}$ & & $\mathbf{2 0 0 5}$ & Relativa (\%) \\
\cline { 2 - 3 } \cline { 5 - 6 } 0 a 19 & 0,63 & 0,52 & & 6,77 & $\mathbf{2 0 1 6}$ \\
20 a 59 & 4,11 & 2,81 & & 44,14 & 7,16 \\
60 e mais & 4,57 & 3,89 & & 49,08 & 38,97 \\
Diferencial & 9,32 & 7,22 & & 100,00 & 53,87 \\
\hline
\end{tabular}

Fonte: Sistema de Informações sobre Mortalidade; estimativas preliminares efetuadas em estudo patrocinado pela Ripsa; estimativas preliminares elaboradas pelo Ministério da Saúde/SVS/CGIAE.

Nota: Os dados foram obtidos no Datasus.

\section{Padrão das causas de óbito no diferencial de mortalidade entre os sexos}

A estrutura etária da mortalidade e o padrão das causas de óbito são dimensões fundamentais para entender e complementar a análise das diferenças de longevidade entre homens e mulheres. Esses padrões permitem identificar os grupos etários e as causas de morte que mais contribuíram para o diferencial em um ano e entre diferentes anos, no sentido de aumento ou redução desse indicador. A Tabela 3 apresenta as contribuições absolutas e relativas dos grupos etários e das causas de óbito, para o diferencial na esperança de vida ao nascer por sexo do município de São Paulo, em 2005 e 2016.

Em 2005, o padrão de causas de óbito para explicar o diferencial de mortalidade era caracterizado pela predominância das causas externas - Cap. XX (27,85\%), seguidas pelas doenças do aparelho circulatório - Cap. IX (26,98\%) e neoplasias - Cap. II (12,73\%). Em 2016, as doenças do aparelho circulatório aumentaram sua participação para $30,06 \%$, ao passo que as causas externas explicaram $22,61 \%$ do hiato e as neoplasias $12,37 \%$ (Tabela 3 ).

Contudo, o padrão de mortalidade por causas varia com a idade, o que levanta a importância de se analisar a contribuição das causas de óbitos dentro de cada grupo etário. Valores positivos indicam que a causa de morte contribuiu para aumentar o diferencial, ou seja, a taxa específica de mortalidade pela causa analisada foi maior para os homens do que para as mulheres do grupo etário. Valores negativos, por sua vez, indicam que a causa de óbito em questão contribuiu para reduzir a diferença entre as esperanças de vida ao nascer feminina e masculina. 
TABELA 3

Contribuição absoluta e relativa dos grupos etários e causas de morte para o diferencial na esperança de vida ao nascer por sexo

Município de São Paulo - 2005-2016

\begin{tabular}{|c|c|c|c|c|c|c|c|c|}
\hline $\begin{array}{c}\text { Grupos etários } \\
\text { (em anos) }\end{array}$ & Cap. I & Cap. II & Cap. IX & Cap. X & Cap. XI & Cap. XX & $\begin{array}{l}\text { Demais } \\
\text { causas }\end{array}$ & Total \\
\hline 2005 & \multicolumn{8}{|c|}{ Absoluta (em anos) } \\
\hline 0 a 19 & 0,01 & 0,02 & 0,01 & 0,02 & 0,01 & 0,44 & 0,13 & 0,63 \\
\hline 20 a 59 & 0,33 & 0,09 & 0,75 & 0,18 & 0,48 & 1,95 & 0,32 & 4,11 \\
\hline 60 e mais & 0,11 & 1,08 & 1,75 & 0,76 & 0,30 & 0,21 & 0,37 & 4,57 \\
\hline \multirow[t]{2}{*}{ Total } & 0,45 & 1,19 & 2,51 & 0,96 & 0,79 & 2,59 & 0,82 & 9,32 \\
\hline & \multicolumn{8}{|c|}{ Relativa (\%) } \\
\hline 0 a 19 & 0,06 & 0,17 & 0,16 & 0,18 & 0,08 & 4,72 & 1,40 & 6,77 \\
\hline 20 a 59 & 3,53 & 1,01 & 8,08 & 1,97 & 5,19 & 20,89 & 3,48 & 44,14 \\
\hline 60 e mais & 1,19 & 11,55 & 18,74 & 8,19 & 3,25 & 2,24 & 3,93 & 49,08 \\
\hline Total & 4,78 & 12,73 & 26,98 & 10,35 & 8,51 & 27,85 & 8,81 & 100,00 \\
\hline 2016 & \multicolumn{8}{|c|}{ Absoluta (em anos) } \\
\hline 0 a 19 & 0,01 & 0,01 & 0,03 & 0,03 & 0,00 & 0,32 & 0,12 & 0,52 \\
\hline 20 a 59 & 0,21 & $-0,03$ & 0,71 & 0,26 & 0,31 & 1,12 & 0,23 & 2,81 \\
\hline 60 e mais & 0,08 & 0,92 & 1,43 & 0,64 & 0,29 & 0,19 & 0,33 & 3,89 \\
\hline \multirow[t]{2}{*}{ Total } & 0,31 & 0,89 & 2,17 & 0,93 & 0,60 & 1,63 & 0,68 & 7,22 \\
\hline & \multicolumn{8}{|c|}{ Relativa (\%) } \\
\hline 0 a 19 & 0,14 & 0,07 & 0,39 & 0,36 & 0,05 & 4,45 & 1,69 & 7,16 \\
\hline 20 a 59 & 2,97 & $-0,43$ & 9,82 & 3,67 & 4,29 & 15,53 & 3,12 & 38,97 \\
\hline 60 e mais & 1,14 & 12,73 & 19,85 & 8,92 & 4,03 & 2,62 & 4,58 & 53,87 \\
\hline Total & 4,25 & 12,37 & 30,06 & 12,95 & 8,37 & 22,61 & 9,40 & 100,00 \\
\hline
\end{tabular}

Fonte: Sistema de Informações sobre Mortalidade; estimativas preliminares efetuadas em estudo patrocinado pela Ripsa; estimativas preliminares elaboradas pelo Ministério da Saúde/SVS/CGIAE.

Nota: Os dados foram obtidos no Datasus.

CID 10 - Classificação Internacional de Doenças; Cap. I: Algumas doenças infecciosas e parasitárias; Cap. II: Neoplasmas [tumores]; Cap. IX: Doenças do aparelho circulatório; Cap. X: Doenças do aparelho respiratório; Cap. XI: Doenças do aparelho digestivo; Cap. XX: Causas externas de morbidade e de mortalidade.

Os resultados indicam que a contribuição das crianças e jovens, que explicou menos de $10 \%$ do diferencial entre os dois anos, é resultado principalmente do maior risco de morte dos homens por causas externas. 0 mesmo acontece com o grupo etário dos adultos, em que as causas externas são as responsáveis pela maior contribuição para o diferencial, seguidas por doenças do aparelho circulatório e do aparelho digestivo. No entanto, entre os anos estudados, houve redução da participação das causas externas e aumento das doenças do aparelho circulatório neste grupo etário. Além disso, é importante observar que, em 2016, as mulheres adultas apresentaram uma taxa específica de mortalidade por neoplasias maior do que a dos homens, o que levou a uma contribuição negativa para o diferencial (Tabela 3).

Os óbitos por causas externas explicaram $20,89 \%$ da contribuição dos adultos ao hiato na expectativa de vida ao nascer entre os sexos, em 2005, e 15,53\%, em 2016. Entre os idosos, os agravos crônicos assumiram boa parte da contribuição para o diferencial. Em 2005, a desvantagem masculina nas doenças do aparelho circulatório, neoplasias e doenças 
do aparelho respiratório foi responsável por 3,58 anos $(78,39 \%)$ da contribuição de 4,57 anos feita pelos idosos ao diferencial. Em 2016, essa contribuição reduziu-se para 77,03\%. As doenças do aparelho circulatório explicaram 18,74\% da contribuição dos idosos, em 2005 , e $19,85 \%$, em 2016 . Ademais, fica evidente a pequena participação das doenças infecciosas e parasitárias em todos os grupos etários para explicar o hiato na esperança de vida ao nascer (Tabela 3).

\section{Discussão}

O município de São Paulo experimentou redução dos seus níveis de mortalidade entre 2005 e 2016, acarretando aumento da esperança de vida ao nascer de homens e mulheres. Assim como na maioria dos países (PATTON et al., 2009), a esperança de vida ao nascer feminina superou a masculina, produzindo o diferencial de mortalidade entre os sexos. Todavia, o ganho sobre o indicador de 2005 foi maior para os homens, o que levou a uma diminuição dessa diferença em 1,30 ano, passando de 8,57 para 7,27 anos. Dessa forma, o município parece continuar seguindo a tendência de redução do diferencial, observada nos países desenvolvidos desde 1970 (GLEl; HORIUCH, 2007; ROGERS et al., 2010; SUNDBERG, 2018) e no município de São Paulo desde 1990 (SIVIERO et al., 2011). Nos países desenvolvidos, a adoção de comportamentos mais preventivos pelos homens e o aumento do tabagismo entre as mulheres (PAMPEL, 2005; PRESTON; WANG, 2006; HEMSTROM, 2016), além da importância de outros fatores não biológicos, como a obesidade, renda, etnia, status socioeconômico (LUY; GAST, 2014; LUY; WEGNER-SIEGMUND, 2015; SUNDBERG et al., 2018), têm sido vistos como vias para a contínua diminuição do diferencial de mortalidade entre os sexos.

No que diz respeito ao padrão etário da mortalidade entre os sexos, observou-se desvantagem masculina em todas as idades, representada pela razão de sexo entre as taxas específicas de mortalidade. Nos dois anos, é possível observar um pico nas idades jovens, o que sugere que a maior sobremortalidade masculina encontra-se nesses grupos etários. Esse comportamento pode ser associado às mortes por causas externas, especialmente homicídios e violências, que atingem prioritariamente os homens jovens e adultos (MATOS; MARTINS, 2013). Contudo, a razão entre as taxas específicas de mortalidade é uma medida de risco relativo, que tem a limitação de não considerar a participação do grupo etário no diferencial. Desse modo, uma razão elevada não significa, necessariamente, que esse grupo etário está entre os que mais contribuem para a formação do diferencial. Nesse contexto, encontra-se a importância de examinar o quanto cada faixa etária explica a diferença entre as esperanças de vida ao nascer feminina e masculina, que está mais relacionada à distribuição do número de óbitos por idade do que ao risco de morte propriamente dito (GLEl, 2005).

0 mesmo comportamento foi revelado pelos resultados desse estudo para o município de São Paulo, no qual a razão nas idades jovens foi alta, mas relativamente poucas pessoas morreram nessas idades, o que resultou em uma contribuição reduzida para o diferencial. 
De fato, o grupo etário dos idosos, que experimentou razões substancialmente menores do que o dos jovens, explicou a maior parte do diferencial. Esse resultado pode ser relacionado a duas tendências observadas nos países desenvolvidos: a concentração dos óbitos nas idades mais avançadas (GLEl, 2005); e a população idosa como responsável pelas maiores contribuições para o hiato na esperança de vida ao nascer (YANG et al., 2012; LE et al., 2015; SUNDBERG et al., 2018).

Em relação ao padrão de causas de óbito, as doenças do aparelho circulatório, as neoplasias e as causas externas foram predominantes na composição do diferencial, enquanto as doenças infecciosas e parasitárias tiveram a menor participação no hiato. Esse comportamento pode representar o avanço da transição epidemiológica no município, com as doenças não transmissíveis e causas externas assumindo proporção importante entre as principais causas de morte, em oposição à menor participação de doenças transmissíveis (PRATA, 1992). Além disso, entre 2005 e 2016, houve uma redução da contribuição das causas externas para o hiato e um aumento das doenças do aparelho circulatório e neoplasias. De acordo com outros estudos, o mesmo tem sido observado nos países desenvolvidos (YANG et al., 2012; LE et al., 2015).

A análise do padrão de causas de óbito em conjunto com a estrutura etária permite entender o quanto cada causa contribui para aumentar ou reduzir a participação dos grupos etários no diferencial. Dessa forma, os resultados apontam que, mesmo que as causas externas estejam entre as que mais contribuem para explicar o diferencial no padrão de causas de óbito, no grupo etário dos idosos (que tem uma contribuição importante para o hiato entre os sexos na mortalidade) sua participação foi pequena. As doenças típicas da população idosa, como as do aparelho circulatório, neoplasias e do aparelho respiratório, foram mais importantes na contribuição desse grupo para o hiato na esperança de vida ao nascer.

A vantagem das mulheres no processo de redução da mortalidade pode ser vista em praticamente todas as idades e causas de morte, com exceção das demais causas de óbito, no grupo dos idosos, e das neoplasias, no grupo etário de 20 a 59 anos. Nessas faixas de idade, o risco de morte experimentado pelas mulheres foi maior do que o dos homens, reduzindo o hiato na esperança de vida ao nascer entre os sexos. Finalmente, os achados evidenciam que a participação dos grupos etários de crianças, jovens e adultos na composição do diferencial deve-se, principalmente, às causas externas. A menor participação dessas causas na contribuição dos idosos não só evidencia como o comportamento mais arriscado dos homens varia com a idade, mas também é uma informação relevante para o desenvolvimento de estratégias de prevenção e redução da sobremortalidade masculina.

Segundo Vallin (2004), as mudanças no diferencial como resultado da luta contra doenças não transmissíveis dependem não somente de procedimentos médicos sofisticados, mas também da participação ativa de cada indivíduo. Ao contrário da redução das doenças transmissíveis alcançada com o uso de técnicas simples e eficazes, como as vacinas e antibióticos, as doenças não transmissíveis dependem, além de tratamentos específicos, de ações individuais de prevenção. Assim, um dos motivos que explicariam a vantagem 
da mulher no diferencial, no contexto da transição epidemiológica, seria a consciência feminina de necessidade de mudança de comportamento e prevenção. Segundo o autor, essa tendência levou as mulheres a experimentarem uma redução da mortalidade cardiovascular mais rápida do que os homens, podendo ser vista como uma via de mudanças no hiato de esperança de vida ao nascer entre os sexos.

Nossos resultados indicam um caminho para o diferencial, mas precisam ser mais aprofundados. Estudo realizado para a Suécia, entre 1997 e 2014 (SUNDBERG et al., 218), traz resultados importantes. Segundo os autores, a redução no diferencial entre os sexos na esperança de vida nesse período resultou de três padrões distintos: a mortalidade masculina diminuiu mais rapidamente do que a feminina; para algumas causas, a mortalidade masculina diminuiu, enquanto a feminina aumentou; e a mortalidade por outras causas aumentou para ambos os sexos, mas de forma mais intensa para as mulheres. No caso específico da Suécia, o diferencial está concentrado entre os idosos e essa concentração envelheceu de 1997 para 2014. No que diz respeito às causas, a queda da mortalidade por doenças circulatórias explicou $70 \%$ da redução do diferencial, especialmente as doenças isquêmicas do coração. Os autores defendem que o tabagismo pode ter um papel importante nessa redução, no entanto, a crescente prevalência da obesidade poderia ter um efeito neutralizador. Diante da concentração do diferencial entre os idosos, no presente estudo, é importante entender se o mesmo comportamento também é observado no município de São Paulo. Ademais, abrir os grupos de causa que contribuíram mais para o diferencial também lançará luz nessa discussão.

Uma vez que os idosos têm um papel importante no hiato por sexo na mortalidade, outras abordagens podem ser adotadas para compreender mais profundamente a questão. Lenart et al. (2019) estudaram a questão sob uma outra ótica: procuraram entender se o fato de as mulheres viverem mais, em média, reflete em um envelhecimento mais lento por parte delas em relação aos homens. Por meio de dados do Human Mortality Database para 13 países de alta renda e de modelos paramétricos e não paramétricos, os resultados sugerem que os homens envelhecem mais rápido do que as mulheres, no entanto, as diferenças são pequenas. Ademais, as taxas de mortalidade masculina se comportam como as das mulheres oito anos mais velhas.

A compressão da mortalidade é outra abordagem que poderá contribuir no entendimento dos achados desse estudo. Ela é caracterizada pelo aumento da idade média à morte em conjunto com a redução da dispersão dos óbitos ao redor dessa idade (FRIES, 1980). Para o estado de São Paulo, entre 1980 e 2005, Gonzaga et al. (2009) encontraram uma variabilidade da idade à morte para as mulheres significativamente menor do que para os homens, sugerindo que os ganhos de sobrevivência nas idades mais avançadas foram maiores para as mulheres. Kanso (2014) observou a mesma tendência para o Brasil, entre 1980 e 2010.

Outra dimensão que pode ser investigada é a interação entre idade e causas de morte, a qual não foi considerada neste estudo. A questão relacionada ao uso de efeitos de interação 
e de efeitos principais em um mesmo modelo é debatida na literatura e em grupos de discussão acadêmicos e, embora seja uma dimensão importante a ser considerada, existem restrições no seu uso indiscriminado. No caso específico de diferenciais de mortalidade e esperança de vida, diversos métodos de decomposição estão disponíveis na literatura internacional, alguns deles com termo de interação. Castro (2001) compara alguns desses métodos, contínuos e discretos, fazendo um exercício com dados de mortalidade de diferentes regiões do Brasil, entre 1940 e 1990, apontando os pontos fortes, fragilidades e limitações de cada um. Ponnapalli (2005) faz um exercício metodológico semelhante e conclui que todas as técnicas, das mais simples às mais complexas, utilizadas em sua forma modificada chegarão a resultados semelhantes. Trabalhos futuros poderão aprofundar essa temática para dados mais recentes no Brasil.

\section{Considerações finais}

A população masculina experimentou mortalidade superior à feminina em todos os grupos etários no município de São Paulo, assim como as esperanças de vida ao nascer e em outras idades foram maiores entre as mulheres. 0 diferencial de nível de mortalidade entre os sexos apresentou um comportamento decrescente entre os anos, acompanhando a tendência observada nos países desenvolvidos. Mesmo diante de elevadas razões de sexo entre as taxas específicas de mortalidade nas idades jovens, os idosos foram responsáveis pelas maiores contribuições para o diferencial de mortalidade entre os sexos. A análise do padrão de causas de óbito mostrou que as doenças do aparelho circulatório, neoplasias e doenças do aparelho respiratório foram as que apresentaram as contribuições mais expressivas para o diferencial, nas idades avançadas.

A estimativa de mortalidade e a avaliação de seus diferenciais podem auxiliar na formulação de políticas de saúde direcionadas à redução das desigualdades em grupos populacionais específicos. Dessa forma, um estudo posterior com a análise mais específica das contribuições dos óbitos, desagregando as categorias CID 10, poderá auxiliar na compreensão do diferencial de mortalidade entre os sexos no município.

\section{Referências}

CARVALHO J. A. M.; SAWYER, D. O.; RODRIGUES, R. N. Introdução a alguns conceitos básicos e medidas em demografia. 2. ed. São Paulo: Abep, 1994.

CASE, A.; PAXSON, C. Sex differences in morbidity and mortality. Demography, v. 42, n. 2 , p. 189-214, 2005 .

CHOR, D.; DUCHIADE, M. P.; JOURDAN, A. M. F. Diferencial de mortalidade em homens e mulheres em localidade da região Sudeste, Brasil - 1960, 1970 e 1980. Revista de Saúde Pública, v. 26, n. 4, p. 246-255, 1992.

BRASIL. Ministério da Saúde. Datasus. Mortalidade. Disponível em: 〈http://www2.datasus.gov. br/DATASUS/index.php?area=0205〉. Acesso em: 23 jan. 2018. 
BRASIL. Ministério da Saúde. Datasus. População residente. Disponivel em: 〈http://www2. datasus.gov.br/DATASUS/index.php?area=0206>. Acesso em: 23 jan. 2018.

CASTRO M. C. Changes in mortality and life expectancy: some methodological issues. Mathematical Population Studies, v. 9, n. 3-4, p. 181-208, 2001.

CHISUMPA, H. V.; ODIMEGWU, C. W. Decomposition of age and cause-specific adult mortality contributions to the gender gap in life expectancy from census and survey data in Zambia. Population Health, v. 5, p. 218-226, 2018.

FERREIRA, C. E. C.; CASTIÑEIRAS, L. L. O aumento da violência entre jovens adultos e as transformações no padrão de mortalidade paulista. Boletim Como Vai? População Brasileira, v. 1, n. 3, p. 6-12, 1996a.

FERREIRA, C. E. C.; CASTIÑEIRAS, L. L. O rápido aumento da mortalidade dos jovens adultos em São Paulo: uma trágica tendência. São Paulo em Perspectiva, v. 10, n. 2, p. 34-41, 1996b.

FRIES, J. F. Aging, natural death, and the compression of morbidity. The New England Journal of Medicine, n. 303, p. 130-5, 1980.

FUNDAÇÃO SISTEMA ESTADUAL DE ANALISE DE DADOS - SEADE. Esperança de vida aumenta e diferença entre gêneros diminui - Queda de homicídios de jovens poupa vidas e explica avanço masculino. SP Demográfico, ano 7, n. 4, 2006.

GEE, E. M. Gender and death. Encyclopedia of death and dying. New York: Macmillan Reference, 2002. Disponível em: 〈http://www.deathreference.com/En-Gh/Gender-and-Death.html〉. Acesso em: 16 fev. 2018.

GLEI, D. A. The sex gap in mortality: historical patterns across twenty-four countries. Tours, France: International Union for the Scientific Study of Population, 2005. (Paper presented at Section 203: Gender, Health, and Mortality).

GLEI, D. A.; HORIUCH, S. The narrowing sex differential in life expectancy in high-income population: effects of differences in the age pattern of mortality. Population Studies, v. 61, n. 2, p. 141-159, 2007.

GONZAGA, M. R.; QUEIROZ, B. L.; MACHADO, C. J. Compressão da mortalidade: um estudo da variabilidade da idade à morte na população do Estado de São Paulo, Brasil, 1980-2005. Cadernos de Saúde Pública, v. 25, n. 7, p. 1475-1485, jul. 2009.

HEMSTROM, O. Changes in the gender gap in life expectancy in Sweden: a cohort analysis with the most recent trends. In: DINGES. M.; WEIGL, A. (ed.). Gender-specific life expectancy in Europe 1850-2010. Franz Steiner Verlag, 2016.

KALBEN, B. B. Why men die younger: causes of mortality differences by sex. North American Actuarial Journal, v. 4, n. 4, p. 83-111, 2000.

KANSO, S. Compressão da mortalidade no Brasil. In: CAMARANO, A. A. (org.). Novo regime demográfico: uma nova relação entre população e desenvolvimento? Rio de Janeiro: Ipea, 2014. p. 155-175.

LANGFORD, C.; STOREY, P. Sex differentials in mortality early in the twenty century: Sri Lanka and India compared. Population and Development Review, v. 19, n. 2, p. 263-282, 1993.

LE, Y. et al. The changing gender differences in life expectancy in Chinese cities 2005-2010. Plosone, v. 10, n. 4, 2015.

LENART, P.; KURUCZOVA, D.; JOSHI, P. K.; BIENERTOVÁ-VAŠKŮ, J. Male mortality rates mirror mortality rates of older females. Scientific Reports, v. 9, n. 1, Jul. 2019. DOI: 10.1038/s41598019-47111-w. 
LUY, M.; GAST, K. Do women live longer or do men die earlier? Reflections on the causes of sex differences in life expectancy. Gerontology, n. 60, p. 143-153, 2014.

LUY, M.; WEGNER-SIEGMUNDT, C. The impact of smoking on gender differences in life expectancy: more heterogeneous than often stated. The European Journal of Public Health, v. 25, n. 4, p. 706-710, 2015.

MATOS, K. F.; MARTINS, C. B. de G. Mortalidade por causas externas em crianças, adolescente e jovens: uma revisão bibliográfica. Revista Espaço para a Saúde, v. 14, n. 1-2, p. 82-93, 2013.

OHADIKE, P. O. Evolving indications of mortality differentials by sex in Africa. In: LOPEZ, A. D.; RUZICKA, L. T. Sex differentials in mortality: trends, determinants and consequences. Canberra, Australia: Australian National University, 1983.

PAMPEL, F. C. Cigarette use and the narrowing sex differential in mortality. Population and Development Review, v. 28, n. 1, p. 77-104, 2002.

PAMPEL, F. C. Forecasting sex differences in mortality in high income nations: the contribution of smoking. Demographic Research, v. 13, n. 18, p. 455- 484, 2005.

PAMPEL, F. C. Global patterns and determinants of sex differences in smoking. International Journal of Comparative Sociology, v. 47, n. 6, p. 466-48, 2006.

PATTON, G. C. et al. Global patterns of mortality in young people: a systematic analysis of population health data. The Lancet, n. 374, p. 881-892, 2009.

PONNAPALLI, K. M. A comparison of different methods for decomposition of changes in expectation of life at birth and differentials in life expectancy at birth. Demographic Research, v. 12, n. 7, p. 141-172, 2005.

PRATA, P. R. A transição epidemiológica no Brasil. Cadernos de Saúde Pública, v. 8, n. 2, p.168175, 1992.

PRESTON, S. H.; HEUVELINE, P.; GUILLOT, M. Demography: measuring and modeling population process. Massachusets: Blackweil Publishers, 2001.

PRESTON, S. H.; WANG, H. Sex mortality differences in the United States: the role of cohort smoking patterns. Demography, v. 43, n. 4, p. 631-646, 2006.

ROGERS, R. G. et al. Social, behavioral, and biological factors, and sex diferences in mortality. Demography, v. 47, n. 3, p. 555-578, 2010.

SHRESTHA, L. Population aging in developing countries. Health Affairs, v. 19, n. 3, p. 204-212, 2000.

SIMÕES, C. C. S. Perfis de saúde e mortalidade no Brasil: uma análise de seus condicionantes em grupos populacionais específicos. Brasília: Opas, 2002.

SIVIERO, P. C. L. et al. Diferenciais de mortalidade: níveis e padrões segundo o sexo no município de São Paulo de 1920 a 2005. Revista Brasileira de Estudos de População, v. 28, n. 2, p. 283301, 2011.

SIVIERO, P. C. L. Níveis e padrões do diferencial de mortalidade por sexo no município de São Paulo, 1920-2005. Dissertação (Mestrado) - Centro de Desenvolvimento e Planejamento Regional (Cedeplar), Universidade Federal de Minas Gerais (UFMG), Belo Horizonte, 2009.

SUNDBERG, L.; AGAHI, N.; FRITZELL, J.; FORS, S. Why is the gender gap in life expectancy decreasing? The impact of age- and cause-specific mortality in Sweden 1997-2014. International Journal of Public Health, v. 63, n. 6, p. 673-81, 2018. 
TROVATO, F. Narrowing sex differential in life expectancy in Canada and Austria: comparative analysis. In: VIENNA INSTITUTE OF DEMOGRAPHY. Vienna yearbook of population research. Vienna, Austria: Vienna Institute of Demography, 2005. p. 17-52.

UNITED NATIONS, Department of Economic and Social Affairs, Population Division. World population prospects: the 2017 revision, key findings and advance tables. New York: United Nations, 2017.

UNITED NATIONS. Mortality and the demographic impact of HIV/Aids. World population prospects: the 2004 revision. [S.I.]: United Nations, 2005.

UNITED NATIONS. Mortality and the demographic impact of HIV/Aids. World population prospects: the 2006 revision. [S.I.]: United Nations, 2007.

UNITED NATIONS. Model life tables for developing countries. New York: United Nations, 1982.

VALLIN, J. Mortalidade, sexo e gênero. In: PINNELLI, A. (org.). Gênero nos estudos de população. Campinas: Abep, 2004. p. 15-54 (Coleção Demographicas, 2).

VALLIN, J. Sex patterns of mortality: a comparative study of model life tables and actual situations with special reference to the cases of Algeria and France. In: LOPEZ, A. D.; RUZICKA, L. T. Sex differentials in mortality: trends, determinants and consequences. Camberra: Australian National University, 1983.

WISSER, O.; VAUPEL, J. W. The sex differential in mortality: a historical comparison of the adult-age pattern of the ratio and the difference. Rostock: Max Planck Institute for Demographic Research, 2014. (MPIDR Working Paper).

YANG, S. et al. The changing gender differences in life expectancy in Korea 1970-2005. Social Science \& Medicine, v. 75, n. 7, p. 1280-1287, 2012.

YOKOTA, R. T. et al. Contribution of chronic conditions to gender disparities in health expectancies in Belgium, 2001, 2004 and 2008. European Journal of Public Health, v. 29, n. 1, p. 1-6, 2018.

\section{Sobre as autoras}

Pamila Cristina Lima Siviero é doutora e mestre em Demografia pelo Centro de Desenvolvimento e Planejamento Regional (Cedeplar), da Universidade Federal de Minas Gerais (UFMG) e graduada em Ciências Atuariais pela UFMG. Professora do Instituto de Ciências Sociais Aplicadas da Universidade Federal de Alfenas.

Larissa Gonçalves de Souza é doutoranda em Demografia pelo Centro de Desenvolvimento e Planejamento Regional (Cedeplar), da Universidade Federal de Minas Gerais (UFMG), mestre em Estatística Aplicada e Biometria pela Universidade Federal de Alfenas (Unifal-MG) e graduada em Ciências Atuariais pela Unifal-MG. Professora do Instituto de Ciências Sociais Aplicadas da Universidade Federal de Alfenas.

Carla Jorge Machado é PhD em Demografia pela Johns Hopkins University, mestre em Demografia pela Universidade Federal de Minas Gerais (UFMG) e graduada em Ciências Econômicas pela UFMG. Professora associada do Departamento de Medicina Preventiva e Social da Faculdade de Medicina da UFMG. 


\title{
Endereço para correspondência
}

\author{
Pamila Cristina Lima Siviero \\ Avenida Celina Ferreira Otoni, 4000, Bairro Padre Vitor \\ 37048-395 - Varginha-MG, Brasil \\ Larissa Gonçalves de Souza \\ Rua São Paulo, 1190, apt. 102, Centro \\ 30170-131 - Belo Horizonte-MG, Brasil \\ Carla Jorge Machado \\ Av. Professor Alfredo Balena 190, $8^{\circ}$ andar, Centro \\ 30130-100 - Belo Horizonte-MG, Brasil
}

\begin{abstract}
Sex mortality differentials in São Paulo in 2005 and 2016: contribution of age groups and main causes of death
\end{abstract}

The aim of this paper is to analyze the sex mortality differential in the city of São Paulo, in 2005 and 2015 , by analyzing the differential levels and patterns as well as the contribution of different age groups and the main causes of death. The calculated indicators were the differential in life expectancy at birth, the sex ratio between specific mortality rates and the contribution of age groups and causes of death to the differential. The results show that the greater gain of men in life expectancy at birth contributed to the reduction of 1.30 years in the differential between the two years studied. Moreover, despite the high sex ratios among youth-specific mortality rates, older people contributed most to explaining the female advantage in mortality. Finally, circulatory diseases, neoplasms and external causes were responsible for the major contributions to the differential. However, the analysis of the pattern of causes of death together with the age pattern shows that for the elderly, whose contribution is greater to the differential, circulatory diseases, neoplasms and respiratory diseases were more decisive in the formation of the differential.

Keywords: Sex mortality differential. Decomposition. Causes of death.

\section{Resumen}

Diferencias por sexo en la mortalidad en la ciudad de São Paulo en 2005 y 2016: contribución de los grupos de edad y principales causas de muerte

El objetivo de este trabajo es estudiar el diferencial de mortalidad por sexo en la ciudad de São Paulo en 2005 y 2015, analizando los niveles y patrones diferenciales y la contribución de los diferentes grupos de edad y las principales causas de muerte. Los indicadores calculados fueron el diferencial en la esperanza de vida al nacer, la proporción de sexos entre las tasas de mortalidad específicas y la contribución de los grupos de edad y las causas de muerte. Los resultados muestran que la mayor ganancia de los hombres en la esperanza de vida al nacer contribuyó a la reducción de 1,30 años en el diferencial en el período. Además, a pesar de las altas proporciones de sexo entre las tasas de mortalidad específicas de los jóvenes, las personas 
mayores contribuyeron más a explicar la ventaja femenina. Finalmente, las enfermedades circulatorias, las neoplasias y las causas externas fueron responsables de las principales contribuciones al diferencial. Sin embargo, el análisis del patrón de causas de muerte junto con el de edad muestra que, para los ancianos, cuya contribución es mayor al diferencial, las enfermedades circulatorias, las neoplasias y las enfermedades respiratorias fueron más decisivas en su formación.

Palabras clave: Diferencial por sexo en la mortalidade. Descomposición. Causas de muerte.

Recebido para publicação em 06/09/2019

Aceito para publicação em 24/10/2019 


\section{ANEXO}

TABELA A1

Tábua de vida dos homens

Município de São Paulo - 2005

\begin{tabular}{|c|c|c|c|c|c|c|c|c|c|}
\hline$x$ & $n$ & ${ }_{n} m_{x}$ & $\mathrm{n}^{\mathrm{a}_{\mathrm{x}}}$ & ${ }_{n} q_{x}$ & $I_{x}$ & ${ }_{n} d_{x}$ & $n L_{x}$ & $\mathrm{~T}_{\mathrm{x}}$ & $e_{x}$ \\
\hline 0 & 5 & 0,00375 & 2,5 & 0,01856 & 100000 & 1856 & 495360 & 7055533 & 70,56 \\
\hline 5 & 5 & 0,00027 & 2,5 & 0,00135 & 98144 & 132 & 490389 & 6560173 & 66,84 \\
\hline 10 & 5 & 0,00033 & 2,5 & 0,00163 & 98012 & 159 & 489660 & 6069784 & 61,93 \\
\hline 15 & 5 & 0,00179 & 2,5 & 0,00890 & 97852 & 871 & 487083 & 5580124 & 57,03 \\
\hline 20 & 5 & 0,00246 & 2,5 & 0,01224 & 96981 & 1187 & 481938 & 5093041 & 52,52 \\
\hline 25 & 5 & 0,00235 & 2,5 & 0,01169 & 95794 & 1120 & 476169 & 4611103 & 48,14 \\
\hline 30 & 5 & 0,00265 & 2,5 & 0,01319 & 94674 & 1248 & 470248 & 4134934 & 43,68 \\
\hline 35 & 5 & 0,00349 & 2,5 & 0,01730 & 93425 & 1616 & 463086 & 3664687 & 39,23 \\
\hline 40 & 5 & 0,00470 & 2,5 & 0,02324 & 91809 & 2133 & 453713 & 3201600 & 34,87 \\
\hline 45 & 5 & 0,00675 & 2,5 & 0,03320 & 89676 & 2978 & 440936 & 2747887 & 30,64 \\
\hline 50 & 5 & 0,00982 & 2,5 & 0,04792 & 86698 & 4155 & 423106 & 2306951 & 26,61 \\
\hline 55 & 5 & 0,01382 & 2,5 & 0,06678 & 82544 & 5512 & 398939 & 1883846 & 22,82 \\
\hline 60 & 5 & 0,01900 & 2,5 & 0,09070 & 77032 & 6987 & 367692 & 1484906 & 19,28 \\
\hline 65 & 5 & 0,02979 & 2,5 & 0,13863 & 70045 & 9710 & 325950 & 1117214 & 15,95 \\
\hline 70 & 5 & 0,04213 & 2,5 & 0,19059 & 60335 & 11499 & 272926 & 791264 & 13,11 \\
\hline 75 & $\infty$ & 0,09422 & 10,61 & 1,00000 & 48836 & 48836 & 518338 & 518338 & 10,61 \\
\hline
\end{tabular}

Fonte: Sistema de Informações sobre Mortalidade; estimativas preliminares efetuadas em estudo patrocinado pela Ripsa; estimativas preliminares elaboradas pelo Ministério da Saúde/SVS/CGIAE.

TABELA A2

Tábua de vida das mulheres

Município de São Paulo - 2005

\begin{tabular}{cccccccccc}
\hline $\mathbf{x}$ & $\mathrm{n}$ & ${ }_{\mathrm{n}} \mathrm{m}_{\mathbf{x}}$ & $\mathrm{n}_{\mathrm{x}}$ & $\mathrm{n}_{\mathbf{x}}$ & \multicolumn{1}{c}{$\mathrm{l}_{\mathbf{x}}$} & ${ }_{\mathrm{n}} \mathrm{d}_{\mathbf{x}}$ & ${ }_{\mathrm{n}} \mathrm{L}_{\mathbf{x}}$ & $\mathrm{T}_{\mathbf{x}}$ & $\mathrm{e}_{\mathbf{x}}$ \\
\hline 0 & 5 & 0,00320 & 2,5 & 0,01589 & 100000 & 1589 & 496028 & 7987165 & 79,87 \\
5 & 5 & 0,00025 & 2,5 & 0,00123 & 98411 & 121 & 491754 & 7491137 & 76,12 \\
10 & 5 & 0,00024 & 2,5 & 0,00121 & 98290 & 119 & 491155 & 6999382 & 71,21 \\
15 & 5 & 0,00041 & 2,5 & 0,00206 & 98172 & 202 & 490353 & 6508227 & 66,29 \\
20 & 5 & 0,00050 & 2,5 & 0,00252 & 97970 & 247 & 489231 & 6017874 & 61,43 \\
25 & 5 & 0,00063 & 2,5 & 0,00313 & 97723 & 306 & 487848 & 5528644 & 56,57 \\
30 & 5 & 0,00082 & 2,5 & 0,00410 & 97417 & 400 & 486084 & 5040795 & 51,74 \\
35 & 5 & 0,00121 & 2,5 & 0,00603 & 97017 & 585 & 483621 & 4554711 & 46,95 \\
40 & 5 & 0,00197 & 2,5 & 0,00980 & 96432 & 945 & 479794 & 4071090 & 42,22 \\
45 & 5 & 0,00316 & 2,5 & 0,01566 & 95486 & 1495 & 473693 & 3591296 & 37,61 \\
50 & 5 & 0,00458 & 2,5 & 0,02266 & 93991 & 2130 & 464630 & 3117603 & 33,17 \\
55 & 5 & 0,00679 & 2,5 & 0,03339 & 91861 & 3067 & 451638 & 2652973 & 28,88 \\
60 & 5 & 0,00953 & 2,5 & 0,04652 & 88794 & 4131 & 433642 & 2201336 & 24,79 \\
65 & 5 & 0,01555 & 2,5 & 0,07484 & 84663 & 6336 & 407474 & 1767694 & 20,88 \\
70 & 5 & 0,02322 & 2,5 & 0,10972 & 78327 & 8594 & 370148 & 1360219 & 17,37 \\
75 & $\infty$ & 0,07043 & 14,20 & 1,00000 & 69733 & 69733 & 990071 & 990071 & 14,20 \\
\hline
\end{tabular}

Fonte: Sistema de Informações sobre Mortalidade; estimativas preliminares efetuadas em estudo patrocinado pela Ripsa; estimativas preliminares elaboradas pelo Ministério da Saúde/SVS/CGIAE. 
TABELA A3

Tábua de vida dos homens

Município de São Paulo - 2016

\begin{tabular}{rrrrrrrrrr}
\hline $\mathbf{x}$ & $\mathrm{n}$ & ${ }_{\mathrm{n}} \mathrm{m}_{\mathrm{x}}$ & ${ }_{\mathrm{n}} \mathrm{a}_{\mathrm{x}}$ & \multicolumn{1}{c}{$\mathrm{nq}_{\mathbf{x}}$} & \multicolumn{1}{c}{$\mathrm{I}_{\mathrm{x}}$} & \multicolumn{1}{c}{${ }_{\mathrm{n}} \mathrm{d}_{\mathrm{x}}$} & ${ }_{\mathrm{n}} \mathrm{L}_{\mathrm{x}}$ & $\mathrm{T}_{\mathrm{x}}$ & $\mathrm{e}_{\mathrm{x}}$ \\
\hline 0 & 5 & 0,00286 & 2,5 & 0,01421 & 100000 & 1420,77 & 496448,1 & 7349309 & 73,49 \\
5 & 5 & 0,00021 & 2,5 & 0,00103 & 98579,23 & 101,773 & 492641,7 & 6852861 & 69,52 \\
10 & 5 & 0,00028 & 2,5 & 0,00142 & 98477,46 & 139,899 & 492037,5 & 6360219 & 64,59 \\
15 & 5 & 0,00158 & 2,5 & 0,00788 & 98337,56 & 774,793 & 489750,8 & 5868182 & 59,67 \\
20 & 5 & 0,00185 & 2,5 & 0,00922 & 97562,76 & 899,085 & 485566,1 & 5378431 & 55,13 \\
25 & 5 & 0,00158 & 2,5 & 0,00785 & 96663,68 & 759,288 & 481420,2 & 4892865 & 50,62 \\
30 & 5 & 0,00175 & 2,5 & 0,00870 & 95904,39 & 834,38 & 477436 & 4411445 & 46,00 \\
35 & 5 & 0,00223 & 2,5 & 0,01107 & 95070,01 & 1052,58 & 472718,6 & 3934009 & 41,38 \\
40 & 5 & 0,00314 & 2,5 & 0,01556 & 94017,43 & 1462,49 & 466430,9 & 3461290 & 36,82 \\
45 & 5 & 0,00472 & 2,5 & 0,02333 & 92554,93 & 2158,97 & 457377,3 & 2994859 & 32,36 \\
50 & 5 & 0,00747 & 2,5 & 0,03668 & 90395,97 & 3315,92 & 443690 & 2537482 & 28,07 \\
55 & 5 & 0,01108 & 2,5 & 0,05391 & 87080,05 & 4694,1 & 423665 & 2093792 & 24,04 \\
60 & 5 & 0,01649 & 2,5 & 0,07919 & 82385,95 & 6523,74 & 395620,4 & 1670127 & 20,27 \\
65 & 5 & 0,02367 & 2,5 & 0,11173 & 75862,21 & 8475,82 & 358121,5 & 1274507 & 16,80 \\
70 & 5 & 0,03461 & 2,5 & 0,15928 & 67386,39 & 10733 & 310099,5 & 916385,1 & 13,60 \\
75 & $\infty$ & 0,09344 & 10,70 & 1,00000 & 56653,4 & 56653,4 & 606285,7 & 606285,7 & 10,70 \\
\hline
\end{tabular}

Fonte: Sistema de Informações sobre Mortalidade; Estimativas preliminares efetuadas em estudo patrocinado pela Ripsa; estimativas preliminares elaboradas pelo Ministério da Saúde/SVS/CGIAE.

TABELA A4

Tábua de vida das mulheres

Município de São Paulo - 2016

\begin{tabular}{|c|c|c|c|c|c|c|c|c|c|}
\hline$x$ & $n$ & ${ }_{n} m_{x}$ & $\mathrm{n}^{\mathrm{a}} \mathrm{x}$ & $n q_{x}$ & $\mathrm{I}_{\mathrm{x}}$ & ${ }_{n} d_{x}$ & $n L_{x}$ & $\mathrm{~T}_{\mathrm{x}}$ & $e_{x}$ \\
\hline 0 & 5 & 0,0025 & 2,5 & 0,01244 & 100000 & 1244,23 & 496889 & 8071390 & 80,71 \\
\hline 5 & 5 & 0,0002 & 2,5 & 0,00085 & 98755,8 & 84,1641 & 493568 & 7574501 & 76,70 \\
\hline 10 & 5 & 0,0002 & 2,5 & 0,00102 & 98671,6 & 101,125 & 493105 & 7080932 & 71,76 \\
\hline 15 & 5 & 0,0004 & 2,5 & 0,00213 & 98570,5 & 209,705 & 492328 & 6587827 & 66,83 \\
\hline 20 & 5 & 0,0005 & 2,5 & 0,00266 & 98360,8 & 262,014 & 491149 & 6095499 & 61,97 \\
\hline 25 & 5 & 0,0005 & 2,5 & 0,00268 & 98098,8 & 263,24 & 489836 & 5604350 & 57,13 \\
\hline 30 & 5 & 0,0007 & 2,5 & 0,00363 & 97835,5 & 354,984 & 488290 & 5114514 & 52,28 \\
\hline 35 & 5 & 0,0011 & 2,5 & 0,00527 & 97480,5 & 513,74 & 486118 & 4626224 & 47,46 \\
\hline 40 & 5 & 0,0016 & 2,5 & 0,00793 & 96966,8 & 768,569 & 482913 & 4140106 & 42,70 \\
\hline 45 & 5 & 0,0024 & 2,5 & 0,01204 & 96198,2 & 1158,28 & 478095 & 3657193 & 38,02 \\
\hline 50 & 5 & 0,0037 & 2,5 & 0,01809 & 95040 & 1718,87 & 470903 & 3179098 & 33,45 \\
\hline 55 & 5 & 0,0055 & 2,5 & 0,02730 & 93321,1 & 2548,07 & 460235 & 2708195 & 29,02 \\
\hline 60 & 5 & 0,0085 & 2,5 & 0,04151 & 90773 & 3767,86 & 444445 & 2247960 & 24,76 \\
\hline 65 & 5 & 0,0127 & 2,5 & 0,06171 & 87005,2 & 5369,32 & 421603 & 1803514 & 20,73 \\
\hline 70 & 5 & 0,0199 & 2,5 & 0,09461 & 81635,8 & 7723,91 & 388869 & 1381912 & 16,93 \\
\hline 75 & $\infty$ & 0,0744 & 13,44 & 1,00000 & 73911,9 & 73911,9 & 993042 & 993042,2 & 13,44 \\
\hline
\end{tabular}

Fonte: Sistema de Informações sobre Mortalidade; estimativas preliminares efetuadas em estudo patrocinado pela Ripsa; estimativas preliminares elaboradas pelo Ministério da Saúde/SVS/CGIAE. 
TABELA A5

Padrão do diferencial de mortalidade entre os sexos Município de São Paulo - 2005-2016

\begin{tabular}{|c|c|c|c|c|c|c|}
\hline \multirow{3}{*}{$\begin{array}{l}\text { Grupos etários } \\
\text { (em anos) }\end{array}$} & \multirow{2}{*}{\multicolumn{2}{|c|}{$\begin{array}{l}\text { Razão de sexo } \\
\text { entre as taxas }\end{array}$}} & \multicolumn{4}{|c|}{$\begin{array}{c}\text { Contribuição dos grupos etários para o diferencial de } \\
\text { esperança de vida ao nascer }\end{array}$} \\
\hline & & & \multicolumn{2}{|c|}{ Absoluta (em anos) } & \multicolumn{2}{|c|}{ Relativa (\%) } \\
\hline & 2005 & 2016 & 2005 & 2016 & 2005 & 2016 \\
\hline 0 a 4 & 1,17 & 1,14 & 0,20 & 0,13 & 2,12 & 1,85 \\
\hline 5 a 9 & 1,10 & 1,21 & 0,01 & 0,01 & 0,09 & 0,17 \\
\hline 10 a 14 & 1,35 & 1,39 & 0,03 & 0,03 & 0,28 & 0,36 \\
\hline 15 a 19 & 4,34 & 3,71 & 0,40 & 0,35 & 4,28 & 4,79 \\
\hline 20 a 24 & 4,88 & 3,47 & 0,52 & 0,36 & 5,58 & 5,01 \\
\hline 25 a 29 & 3,75 & 2,93 & 0,42 & 0,26 & 4,46 & 3,60 \\
\hline 30 a 34 & 3,23 & 2,40 & 0,40 & 0,23 & 4,26 & 3,19 \\
\hline 35 a 39 & 2,88 & 2,11 & 0,44 & 0,24 & 4,72 & 3,27 \\
\hline 40 a 44 & 2,39 & 1,97 & 0,46 & 0,27 & 4,96 & 3,80 \\
\hline 45 a 49 & 2,14 & 1,95 & 0,52 & 0,35 & 5,63 & 4,90 \\
\hline 50 a 54 & 2,14 & 2,05 & 0,64 & 0,50 & 6,91 & 6,92 \\
\hline 55 a 59 & 2,03 & 2,00 & 0,71 & 0,60 & 7,62 & 8,29 \\
\hline 60 a 64 & 1,99 & 1,95 & 0,76 & 0,69 & 8,15 & 9,56 \\
\hline 65 a 69 & 1,92 & 1,86 & 0,87 & 0,72 & 9,29 & 9,96 \\
\hline 70 a 74 & 1,81 & 1,74 & 0,82 & 0,70 & 8,83 & 9,63 \\
\hline 75 e mais & 1,34 & 1,26 & 2,12 & 1,78 & 22,81 & 24,72 \\
\hline Total & & & & & 100,00 & 100,00 \\
\hline$e_{0}(f e m)-e_{0}(\operatorname{masc})$ & & & 9,32 & 7,22 & & \\
\hline
\end{tabular}

Fonte: Sistema de Informações sobre Mortalidade; estimativas preliminares efetuadas em estudo patrocinado pela Ripsa; Estimativas preliminares elaboradas pelo Ministério da Saúde/SVS/CGIAE.

Nota: Todos os dados foram obtidos no Datasus. 